\title{
VoDSL Information Management for Broadband Communication Network Access
}

\author{
Sarhan M. Musa ${ }^{1,2}$, Cajetan M. Akujuobi², and Nader F. Mir ${ }^{3}$ \\ ${ }^{1}$ Prairie View A\&M Networking Academy (PVNA) \\ ${ }^{2}$ Center of Excellence for Communication Systems Technology Research (CECSTR), Prairie View A\&M University, Texas \\ ${ }^{3}$ Department of Electrical Engineering, San Jose State University, CA
}

\begin{abstract}
This paper presents the information management of Voice over Digital Subscriber line (VoDSL) network architecture with implications for broadband access technology. A baseline test for the ability to support up to eight derived lines on the Integrated Access Device (IAD) for VoDSL by using voice quality transmission is introduced. This scheme carries out voice compression while countinously downloading files. A fixed wireline network test for the broadband access evolution using VoDSL technology has also been presented, which allows service providers to offer multiple telephone lines over a single subscriber access line in addition to high-speed data transmission services. The design of an experimental VoDSL network architecture is presented. The number of supported derived lines in the voice gateway and IAD has been identified based on a variety of speeds and standards for Digital Subscriber Line (DSL). The novel results are presented in this paper.
\end{abstract}

Keywords: Network information management, Voice over Digital Subscriber Line (VoDSL), Digital Subscriber Line (DSL), Integrated Access Device (IAD), broadband access network.

\section{Introduction}

One of the long-term objectives for telecommunications networks in the multimedia services is to support the future of broadband access network services. There is no doubt, today, that the telecommunication of the fixed wireline networks is vastly adopting the VoDSL as a broadband access technology. There has been growing attention and concern in improving the multimedia services by developing DSL technologies that can provide high bit rate communication over existing non-loaded subscriber loops to satisfy the expectations and demands set for in both quantity and quality of services (e.g., voice and data) [1], [2] and [3].

There is now a range of different DSL technologies available for high-speed transmission over twisted pair copper wire, which are appropriate to the requirements of different customers such as: basic access, High bit rate Digital Subscriber Lines (HDSL), Symmetric Digital Subscriber Lines (SDSL), Very High bit rate Digital Subscriber Lines (VDSL), and Asymmetric Digital Subscriber Lines (ADSL). ADSL is used for asymmetric services to residential and Small Office, Home Office (SOHO) customers. It transmits high bit rate data in the down direction from the central office to the subscriber (downstream), with typical bit rates from 1.5 to $8 \mathrm{Mb} / \mathrm{s}$, and lower bit rate data in the reverse (up) direction from the subscriber to the central office (upstream), with bit rates from 64 to $640 \mathrm{~Kb} / \mathrm{s}$ [4] and [5].

VoDSL has been developed with the rapid increase of the Internet and of the data traffic through network convergence of voice and data information [6]. This paper contains novel results that can be used in evaluating VoDSL solutions that offer multiple voice information (including Fax transmission) connections simultaneously with data information onto the high-speed digital line offered by ADSL. The implications of the wireline access for ADSL have been explored, focusing on the emerging VoDSL technologies by using voice qual- 
ity transmission with voice compression while continuously downloading files and stressing on testing to identify the maximum number of supported derived phone lines per ANSI/CSA loops.

The description of the test setup for the network architecture is included in section 2. Section 3 is the overview of the twisted pair and ADSL technique. Section 4 describes the fixed wireline test setup and section 5 shows the results of the tests performed while section 6 is the conclusion.

\section{Description of Information and Network Architecture}

While continuously downloading files, voice quality transmission with voice compression was used to verify the ability to support up to eight derived lines on the IADs for VoDSL. The following DSL service levels were used $640 \mathrm{~K} / 640 \mathrm{~K}, 1.5 \mathrm{M} / 384 \mathrm{~K}, 3.0 \mathrm{M} / 512 \mathrm{~K}$, and $1.5 \mathrm{M} / 256 \mathrm{~K}$ for each ANSI/CSA loops. Figure 1 shows the fixed wireline networks setup for the access evolution of broadband services using VoDSL technology for voice quality transmission. The voice quality transmission with voice compression has been verified as has the maximum number of line connections in compressed mode operation that can be supported without any problems based on Figure 1.

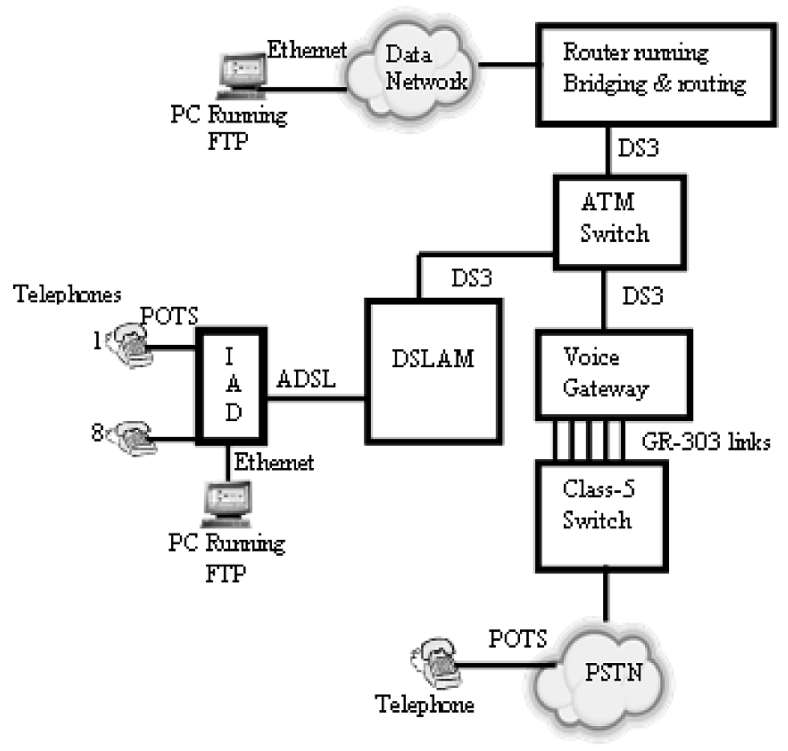

Fig. 1. Voice quality information transmission.
Customers can have multiple IADs based on their needs. Each IAD has 4-8 telephone interfaces plus an Ethernet interface. For this purpose, one IAD was tested based on VoDSL solutions. Eight telephones were connected to the IAD that resides in the customer premises through Plain Old Telephone Service (POTS). Data from the PC source running Files Transfer Protocol (FTP) was provided to the IAD via the Ethernet. IAD interconnects the Customer's Premises Equipment (CPE) and ADSL service. It also converts POTS to Asynchronous Transfer Mode Adaptation Layer type 2 (AAL2). It uses the same Virtual Path Identifier/Virtual Channel Identifier (VPI/VCI) for voice and data. Therefore, these have to be mapped to unique values before transmission on the same pipe out of the Digital Subscriber Line Access Multiplexer (DSLAM). All the voice calls are digitized in the form of AAL2 cells and sent over the same Permanent Virtual Circuit (PVC). AAL2 allows the use of the same VPI/VCI for multiple users. Users do not necessarily have to coexist in an ATM cell. Each cell can carry data from just one user. The data rides separate PVC. Both these PVCs are transported over the ADSL copper connection to a DSLAM. The DSLAM aggregates all the PVCs onto a single connection and sends it to a switch. The switch separates the voice and data calls. The voice PVCs go to a voice gateway and the voice gateway converts the ATM traffic into Time Division Multiplexing (TDM)-analog traffic and interfaces to a Class 5 switch via GR-303 Interfaces. The voice calls go to Public Switched Telephone Network (PSTN) that are connected to POTS, which carries the voice calls to the telephones. On the other hand, the data is sent to a router through Digital Signal-level 3 (DS3 ) and to the Internet then to a PC running FTP through Ethernet.

Confirmation of the dial tone on all the ports of the IAD was needed. This was achieved by connecting phones to the ports and taking them off the hook at the same time and recording the number of phones that simultaneously had dial tones. Calls were made from each port of the IAD to a phone connected to the PSTN and repeated in the same way from a PSTN phone to each port of the IAD. Voice compression was enabled and the number of phones having dial 
tones was verified. These tests performances are based on the Asymmetric Digital Subscriber Line Wireline Simulator (ADSL WLS), which is located between the IAD (at customer) and DSLAM (at Central Office (CO)). ADSL WLS was used to generate and receive the traffic between the customer and $\mathrm{CO}$ after assigning the following loops: CSA loop \#6 (9kft of 26 AWG wire), ANSI loop \#7 with 24 DSL disturbers, ANSI loop \#9 with 24 DSL disturbers, ANSI loop \#13 with 24 DSL disturbers, CSA loop \#4 with 10 ADSL disturbers, 10 HDSL disturbers and 24 DSL disturbers, CSA loop \#6 with 10 ADSL disturbers, 10 HDSL disturbers and 24 DSL disturbers, CSA loop \#8 with 10 ADSL disturbers, 10 HDSL disturbers and 24 DSL disturbers, mid-CSA loop \#6 with 10 T1 disturbers in an adjacent binder group, $9 \mathrm{kft}, 12 \mathrm{kft}$ and $15 \mathrm{kft}$ of $26 \mathrm{AWG}$ cable operating at $6 \mathrm{~dB}$ margin, and null loop.

\section{Overview of Twisted Pair and ADSL Technique}

Two insulated copper wires twisted around each other are called twisted pair. It is a common form of copper cabling used for telephones and data communications. The twisting protects the communications from electromagnetic frequency and radio frequency interference. The cable may be shielded or unshielded. Figure 2 illustrates the typical configuration of a POTS distribution cable in the loop plant (the twisted pair wire infrastructure), that connects customers to the telephone company network. Each pair in the distributed cable may be used like an end user to the $\mathrm{CO}$ for full duplex analog voice transmission. A twisted pair is characterized by the diameter based on the following: its copper wire gauge (such as 24 and 26 which are the most frequently used in the USA), its twist length, and the way it is isolated. Multiple bridge taps are frequently used along each pair near either end or at an intermediate point when the distribution cable is laid [7].

A bridge tap permits all the pairs in the cable to be used or reused to serve any new customer along the cable route. It consists of an unterminated section of Unshielded Twisted Pair (UTP) wiring connected in shunt (which distorts the transmission of electrical signals) to a twisted pair of a distribution cable. The other bridge taps of the same pair remain terminated by an open circuit, when a new end user is connected to the CO on one of the pairs. Signal loss and distortion can exist due to the reflection of signals from the unterminated bridged taps.

ADSL promises enough bandwidth to deliver many types of multimedia services to the end user via the existing local loops. Figure 3 shows how the local-loop bandwidth is used in ADSL line. Figure 4 illustrates how a single twisted pair is used between the $\mathrm{CO}$ and the $\mathrm{CPE}$ with the ADSL modems being located at each end

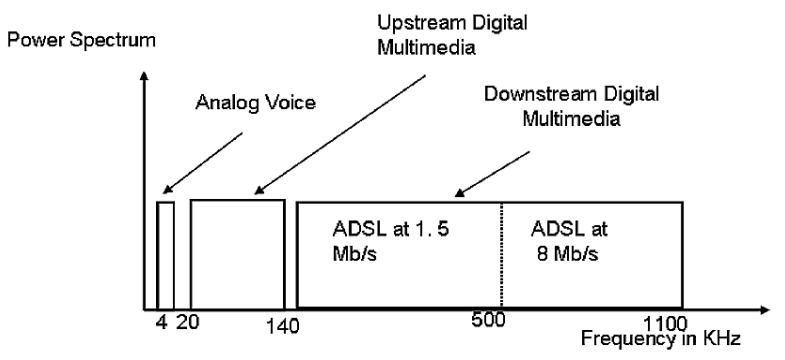

Fig. 3. ADSL frequency spectrum.

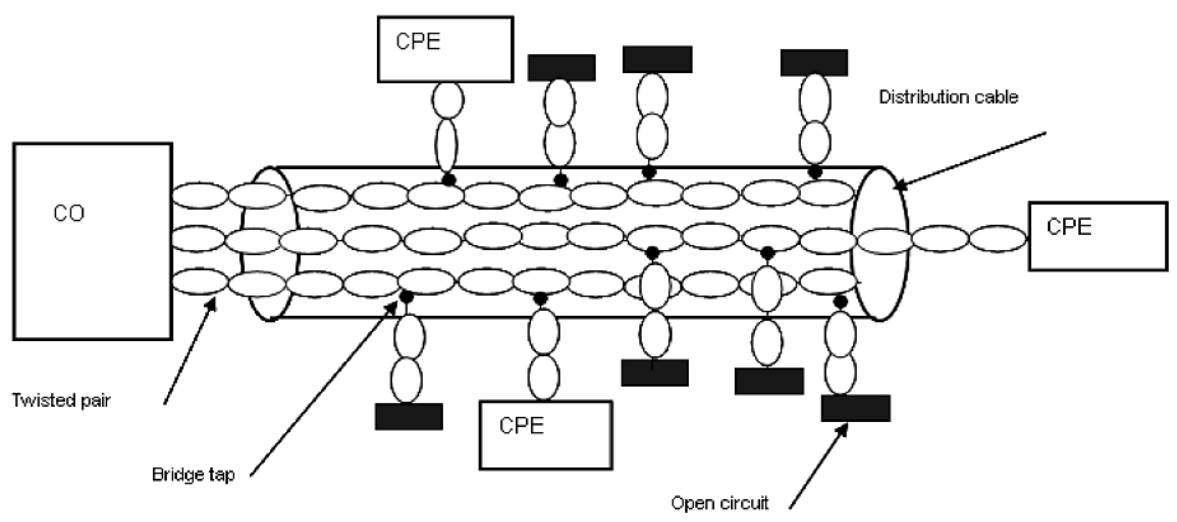

Fig. 2. Configuration of a distribution cable. 


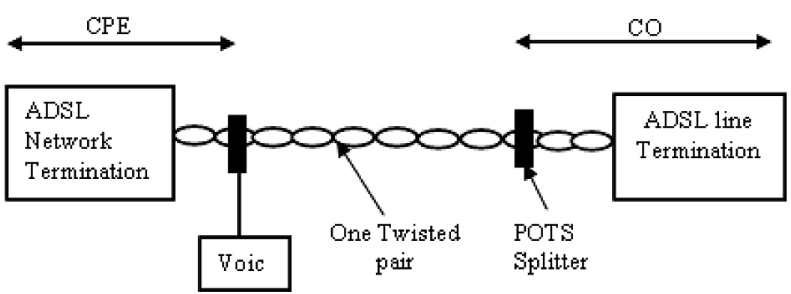

Fig. 4. ADSL access line.

of the line. Today, standard xDSL (a genetic name for a family of digital lines) solutions focus mainly on data services such as high-speed Internet access. In Figure 5 the VoDSL solution based on ADSL access line proposes to take advantage of high data bandwidth available at the ADSL line, to carry one or more voice band services (such as fax, voice band modem, and analog and digital voice) together with the regular data stream.

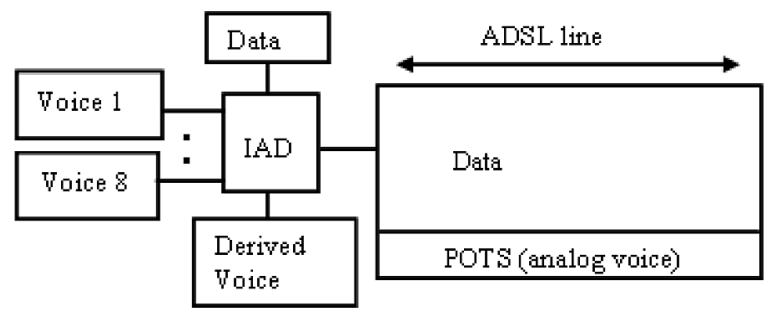

Fig. 5. VoDSL solution based on ADSL access Line.

This can benefit the customers by providing higher flexibility with the following new services: no need to replace existing devices, no waiting for new voice lines (including selfprovisioning of new services), one stop shopping for voice and data services, and lower prices due to bundled offerings, more bandwidth and voice lines over an infrastructure, low costs to the manufacture, and the ability to grow the number of voice lines.

However, there are some limitations of copper lines that reduce the ability to transfer information [8]: First, crosstalk, which is the undesired coupling of a signal from one communication channel to another, occurs when some of the transmissions signal energy leaks from the cable. Crosstalk can be the largest noise impairment in a twisted pair and often considerably reduces transmission performance of DSL systems. Crosstalk on xDSL systems can be divided into two categories; Near End Crosstalk (NEXT) and Far End Crosstalk (FEXT). NEXT occurs between a transmitter and a receiver at

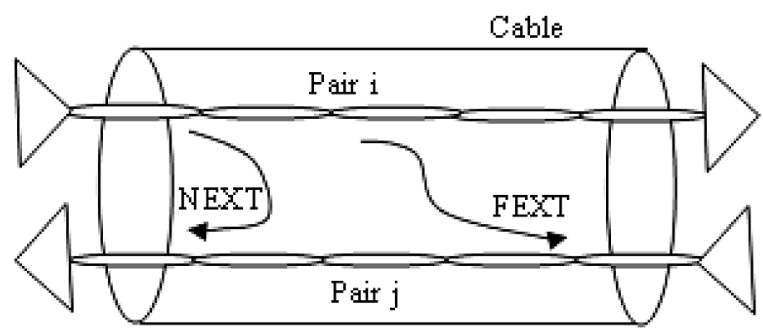

Fig. 6. NEXT and FEXT crosstalk.

the same end of a twisted pair cable. FEXT occurs from adjacent transmitters at the transmitter end that couples to the receiver of another system. Figure 6 shows two types of DSL crosstalk where pairs $\mathrm{j}$ and $\mathrm{i}$ belong to the same distribution cable and operate in full duplex. The NEXT and FEXT models are often used for studies in industry forums as a starting point. The $1 \%$ worst is the case for one type of a source of crosstalk [9]. The Power Spectral Densities (PSDs) of NEXT and FEXT due to one pair-to-pair crosstalk disturber (source) are

$$
\begin{aligned}
& \operatorname{NEXT}(f)=M(f) X_{N} f^{3 / 2} \\
& F E X T(f)=M(f) X_{F} f^{2} d|T(f)|^{2}
\end{aligned}
$$

where $f$ is the frequency, $M(f)$ is the PSD of the distributing signal, $d$ is the loop length, $T(f)$ is the transfer function of the loop, $X_{N}$ and $X_{F}$ are constants determined by measurements [10].

Second, Bridge tap reflections, unterminated bridge taps that are installed on the twisted pair cables transferring DSL signals can result in signal distortion. It comes from the reflections of signal energy reflections off the bridge taps and causes interference at the higher xDSL frequencies. Third, Radio Frequency Interferer (RFI) occurs when electrical signals from other sources, such as radio signals, enter into the transmission line and it reduces the data transmission capacity of the DSL line. Fourth, frequency response depends on a variety of factors including the dimension of the copper wire and insulation type. The frequency response applied to the copper wire pair is directly proportional to the attenuation of the line and the signal energy leakage.

Fifth, audio loading coils are used to adjust the frequency response of the twisted pair line to better transfer audio signals. However, they completely disable the ability of the line to be used for high frequency DSL signals. Sixth, 
line attenuation has two parts, splice attenuation and resistance attenuation. The line splice attenuates the signal and the amount of signal attenuation varies depending on the type of splice such as twist and the amount of corrosion inside the splice. The copper cable resistance depends on the diameter of the cable; it is inversely proportional to the diameter and to the transferred energy to the receiving device. It is directly proportional to the signal energy and is dissipated by the line.

\section{Fixed Wireline Network Setup}

Fax transmission with voice compression was used while simultaneously downloading files to verify the ability to support up to eight derived lines on the IADs for VoDSL. The following DSL service levels were used $-640 \mathrm{~K} / 640 \mathrm{~K}$, $1.5 \mathrm{M} / 384 \mathrm{~K}, 3.0 \mathrm{M} / 512 \mathrm{~K}$, and $1.5 \mathrm{M} / 256 \mathrm{~K}$ for each ANSI/CSA loops as used in the voice quality setup. Figure 7 shows the fixed wireline networks test setup for the access evolution of multimedia services using VoDSL technology for fax transmission. Fax transmission with voice compression has been verified as has the maximum number of line connections in compressed mode operation that can be supported without any problems based on Figure 7.

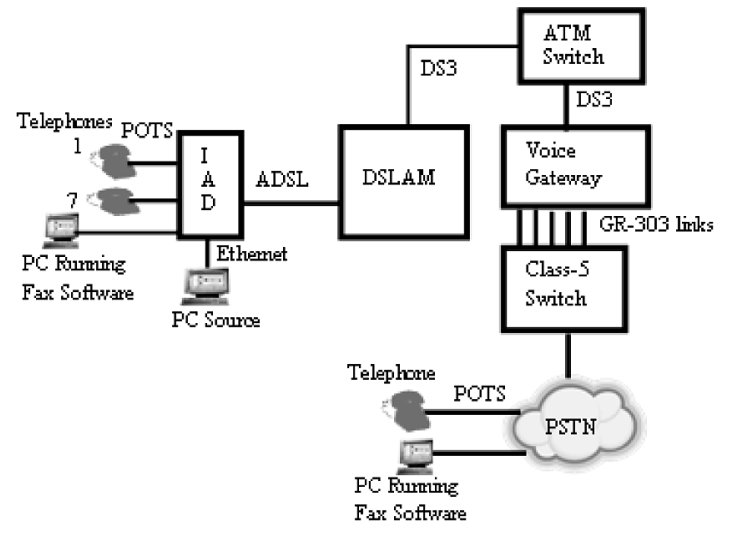

Fig. 7. Fax transmission configuration.

The ATM switch in Figure 7 multiplexes the traffic of voice and data over voice lines. The voice gateway converts the ATM traffic into TDM-analog traffic and interfaces to a Class 5 switch via GR-303 Interfaces. The data goes to PSTN that is connected to POT, which carries the fax to the PC running fax software.
Figure 8 shows some examples of the copper loops impairments as made up of pairs from sections of several cables between the central office and the customer. Because the fax requires 64 kbps operations, if dial tones are detected on eight ports in the fax transmission during voice compression while simultaneously downloading files, it will not be possible for all faxes to be sent at the same time.

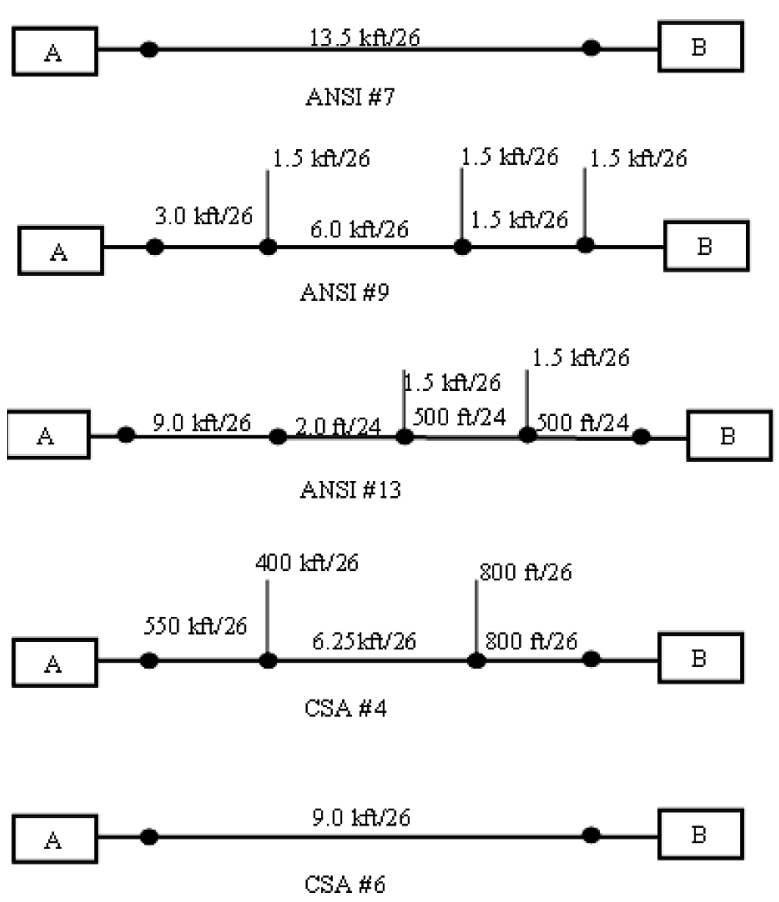

Fig. 8. Examples of ANSI/ CSA loops descriptions.

\section{Results}

During the voice quality transmission testing that included ADSL loops impairments it was found that on certain loops not all eight derived lines were supported. Figure 9 shows the statistics of DSL service levels over different loops versus the number of lines supported at $640 \mathrm{~K} / 640 \mathrm{~K}, 1.5 \mathrm{M} / 384 \mathrm{~K}$ and $3.0 \mathrm{M} / 512 \mathrm{~K}$. At these speeds the DSL service levels have the same results for each ANSI/CSA loop. It could be seen that ANSI loops (\#7, \#9, and \#13) with 24 DSL disturbers support five, three, and five lines respectively. However, the rest of the loops are supported by eight lines.

Figure 10 shows that none of the eleven loops are supported by eight lines at the DSL service 
level $1.5 \mathrm{M} / 256 \mathrm{~K}$. Observations based on figures 9 and 10 reveal that the three ANSI loops (\#7, \#9, and \#13) with 24 DSL disturbers remained constant at every speed. By comparing each DSL service level to the number of lines supported for every loop, the following observation was made: the CSA loop \#6 (9kft of 26 AWG wire) is at all the speeds with the exception of DSL service level of $1.5 \mathrm{M} / 256 \mathrm{~K}$ which supports eight lines. This is also the case for the remaining loops with the exception of the three ANSI loops (\#7, \#9, and \#13), which are supported by not more than five lines. Therefore, it can be concluded that ANSI loops \#7, $\# 9$ and \#13 are not effective loops, they support five lines and less at every speed and this can be seen on the two different plot settings. Also, DSL service level $1.5 \mathrm{M} / 256 \mathrm{~K}$ seems as though it were unable to support more than six lines.

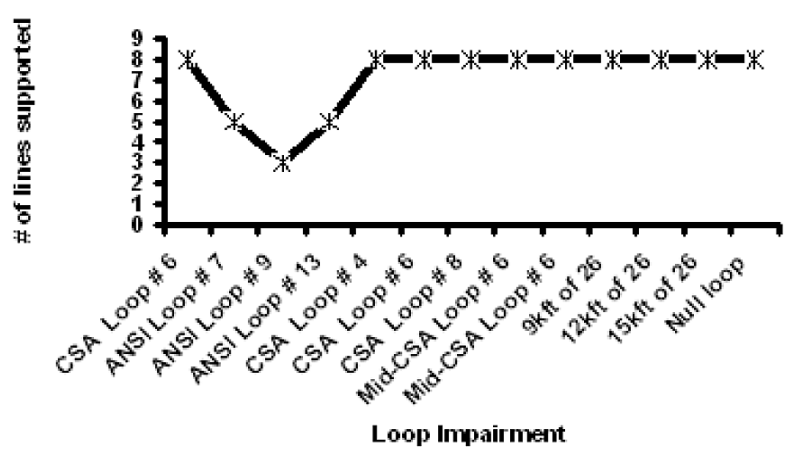

Fig. 9. DSL service level $640 \mathrm{~K} / 640 \mathrm{~K}, 1.5 \mathrm{M} / 384 \mathrm{~K}$ \& $3.0 \mathrm{M} / 512 \mathrm{~K}$.

Finally, it can be justified that CSA loop \#4 with 10 ADSL disturbers, 10 HDSL disturbers and 24 DSL disturbers, CSA loop \#6 with 10 ADSL disturbers, 10 HDSL disturbers and 24 DSL disturbers, CSA loop \#8 with 10 ADSL disturbers, 10 HDSL disturbers and 24 DSL disturbers, CSA loop \#6 for both mid-CSA loops, $9 \mathrm{kft}$ of 26 AWG, 12kft of 26 AWG, 15kft of 26 AWG and the null loop are the best carrier loops to be used within a VoDSL for the maximum number of supported derived phone lines in the voice quality transmission with voice compression while continuously downloading files.

While testing the fax transmission that included ADSL loop impairments it was found that on certain loops not all eight derived lines were supported. The crosstalk noise (NEXT and
FEXT) is one major internal noise factor that affects on the capacity of VoDSL based on ADSL loops impairments. The performance estimation of the actual power sum crosstalk characteristic is different for each individual cable and for each pair combination. At high speed digital transmission, the transfer function interference characteristics of each loop are determined by the length and gauge of the cable segments of which it is composed and by the geometry of connection of those segments. This can also include bridge taps.

Moreover, the loop population can put stress on the transmission system that may limit the performance of the VoDSL technology. Signal reflections (echoes) are a difficult problem in a high-speed transmission on copper loops because of: wide impedance variations from loop to loop, the large difference between transmit, and receive levels on long loops. Impulse noise is a transient waveform that occurs infrequently and has a high peak relative to typical peaks of the background noise. Impulse noise due to loops make (or break) signals and ringing signals, which propagates on adjacent pairs in the destitution cable and is very difficult to predict and analyze [11] and [7].

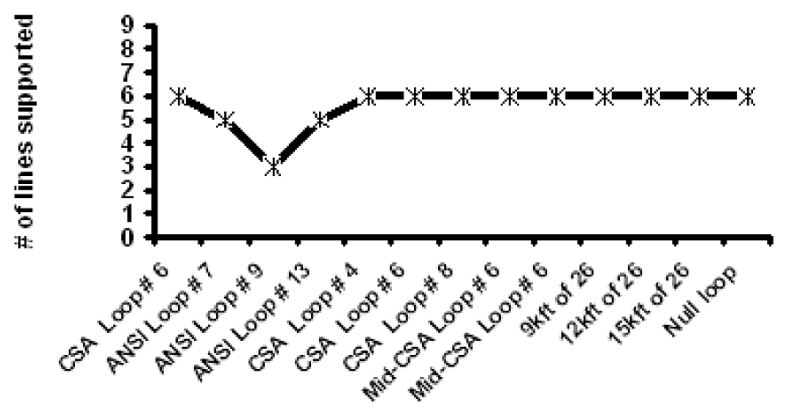

Fig. 10. DSL service level $1.5 \mathrm{M} / 256 \mathrm{~K}$.

Figure 11 shows the statistics of DSL service levels over different loops versus the number of lines supported at $640 \mathrm{~K} / 640 \mathrm{~K}, 1.5 \mathrm{M} / 384 \mathrm{~K}$ and $3.0 \mathrm{M} / 512 \mathrm{~K}$. At these speeds, the DSL service levels have the same results for each ANSI/CSA loop. It could be seen that ANSI loops (\#7, \#9, and \#13) with 24 DSL disturbers are supported by four, two, and five lines respectively. However, the rest of the loops are supported by eight lines. 


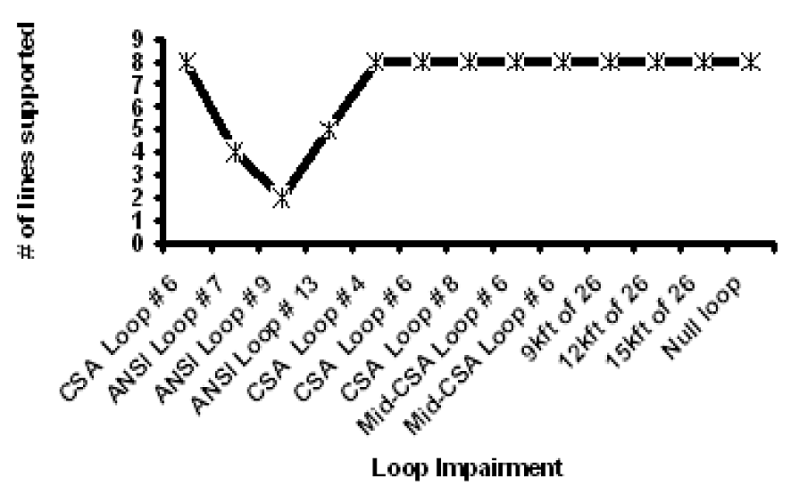

Fig. 11. DSL service level $640 \mathrm{~K} / 640 \mathrm{~K}, 1.5 \mathrm{M} / 384 \mathrm{~K}$ and $3.0 \mathrm{M} / 512 \mathrm{~K}$.

Figure 12 shows that none of the eleven loops are supported by eight lines at the DSL service level $1.5 \mathrm{M} / 256 \mathrm{~K}$. Observations based on Figures 11 and 12 reveal that the three ANSI loops (\#7, \#9, and \#13) with 24 DSL disturbers remained constant at every speed.

These results are based on comparing each DSL service level that represents the number of lines supported vs. loop impairment. In addition, by comparing each loop impairment that represents the number of lines supported vs. DSL speeds.

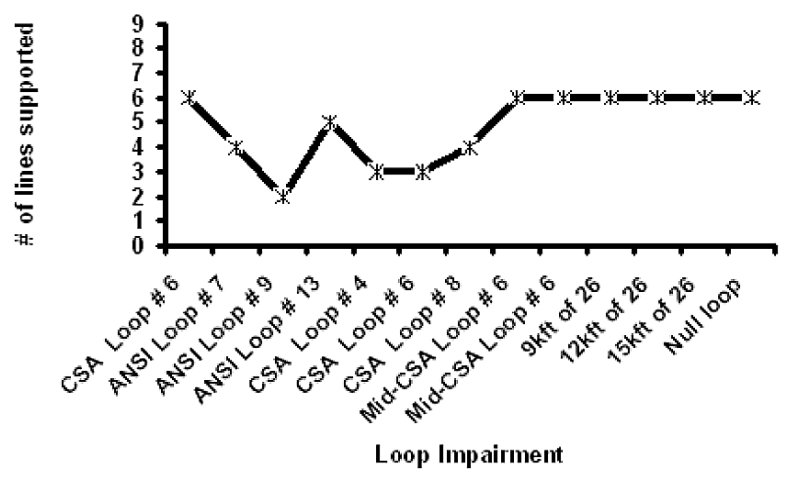

Fig. 12. DSL service level $1.5 \mathrm{M} / 256 \mathrm{~K}$.

By comparing each DSL service level to the number of lines supported for every loop, the following observation was noted: the CSA loop \#6 (9kft of 26 AWG wire) at all speeds with the exception of DSL service level of $1.5 \mathrm{M} / 256 \mathrm{~K}$ supports eight lines.

This also applies to the remaining loops with the exception of the three ANSI loops (\#7, \#9, and \#13), which are supported by not more than five lines. Therefore, it can be concluded that ANSI loops \#7, \#9 and \#13 are not effective loops, they support five lines or less at every speed and this can be seen on the two different plot settings. In addition, DSL service level $1.5 \mathrm{M} / 256 \mathrm{k}$ seems unable to support more than six lines.

Lastly, it can be justified that CSA loop \#6, for mid-CSA loops, 9kft of 26 AWG, $12 \mathrm{kft}$ of 26 AWG, 15kft of 26 AWG and the null loop (of course!) are the best carrier loops to be used within a VoDSL.

\section{Conclusion}

Based on the experimental VoDSL network architecture and the following DSL service levels $640 \mathrm{~K} / 640 \mathrm{~K}, 1.5 \mathrm{M} / 384 \mathrm{~K}, 3.0 \mathrm{M} / 512 \mathrm{~K}$, and $1.5 \mathrm{M} / 256 \mathrm{~K}$ for each ANSI/CSA loop by using voice quality transmission testing with voice compression while continuously downloading files, it was found that on certain loops not all eight derived lines were supported on IADs for VoDSL solution. However, some were supported, as shown in Figures 9 and 10 respectively.

Based on the following DSL service levels $640 \mathrm{~K} / 640 \mathrm{~K}, 1.5 \mathrm{M} / 384 \mathrm{~K}, 3.0 \mathrm{M} / 512 \mathrm{~K}$, and $1.5 \mathrm{M} / 256 \mathrm{~K}$ for each ANSI/CSA loop by using fax transmission with voice compression while simultaneously downloading files, it was found that on certain loops not all eight derived lines were supported on IADs for VoDSL solution. However, some were supported, as shown in Figures 11 and 12 respectively.

\section{References}

[1] D. O'HARE ET AL., Voice over DSL Requirements: MSDN, ADSL Forum 1999-217, 1999.

[2] K. Asatani And Y. Maeda, Access Network Architectural Issues for Future Telecommunication Networks, IEEE Commun. Mag., Vol. 36, No. 8, Aug. 1998.

[3] G. Young, K. T. Foster And J. W. CoOK, Broadband Multimedia Delivery over Copper, BT Tech. J., Vol. 13, No. 4, Oct. 1995.

[4] Network and Customer Installation InterfacesAsymmetric Digital Subscriber Line (ADSL) Metallic Interface, ANSI Standard T1.413, 1995. 
[5] W. Y. CHEN, The Development and Standarization of Asymmetrical Digital Subscriber Line, IEEE Commun. Mag., May 1999, Vol. 37, No. 5, pp. 68-72.

[6] R. FRANZ, Optimal Migration towards Voice/Data Convergence, ICCT 2000 contribution.

[7] V. B. LAWRENCE, L. J. SMithWick AND J. J. WERNER, Broadband access to the home copper, Bell Labs Tech. J., Vol. 1, No., and pp. 100-114, Summer1996.

[8] L. HARTE AND R. KIKTA, Delivering $x D S L$, New York: McGraw-Hill, 2001.

[9] Spectrum Management for Loop Transmission Systems, Committee T1 Standard T1. 417-2001, Jan. 2001.

[10] S. Galli AND K. J. KerPez, Methods of Summing Crosstalk from Mixed Sources-Part I: Theoretical Analysis, IEEE Trans. Commun., Vol. 50, No. 3, March. 2002.

[11] I. KALET AND S. SHAMAI, On the capacity of a twisted-wire pair: Gaussian model, IEEE Trans. Commun., Vol. 38, No. 3, pp. 379-383, Mar. 1990.

Received: January, 2006 Accepted: March, 2006

Contact addresses:

Sarhan M. Musa Prairie View A\&M Networking Academy (PVNA) Center of Excellence for Communication System Technology Research (CECSTR) Prairie View A\&M University Prairie View Texas 77446, USA smmusa@pvamu.edu

Cajetan M. Akujuobi Center of Excellence for Communication Systems Technology Research (CECSTR) Prairie View A\&M University Prairie View

Texas 77446, USA e-mail: cmakujuobi@pvamu.edu

Nader F. Mir Department of Electrical Engineering San Jose State University San Jose CA 95192 USA e-mail: nmir@sjsu.edu
CAJETAN M. AKuJuObi received the B.S. degree from Southern University, Baton Rouge, Louisiana in 1980, and his M.S. and Ph.D. from Tuskegee University, Tuskegee, Alabama and George Mason University, Fairfax, Virginia in 1983 and 1995 respectively, all in electrical engineering. He has also an MBA Degree from Hampton University, Hampton, Virginia, in 1987 in business administration. He is a Professor in the Department of Electrical Engineering and is the founding Director of the Analog \& Mixed Signal (AMS), DSP Solutions and High Speed (Broadband) Communication Programs at Prairie View A\&M University. He is also the founding Director of the Center of Excellence for Communication Systems Technology Research (CECSTR). He has been a member of the IEEE for over 27 years and is one of the founding corporate members of the IEEE Standards Association (IEEESA), Industry Advisory Committee (IAC). He is a senior member of IEEE, senior member of ISA, ASEE, SPIE, and Sigma XI, the Scientific Research Society. His current research interests include wavelet transform-based research, all areas of signal and image processing and communication systems (Broadband Telecommunications). Other research interests are in the areas of DSP Solutions, analog mixed signal system, and control system-based communications. He was a participant and collaborative member of ANSI T1E1.4 Working Group that had the technical responsibility of developing T1.413, Issue 2 ADSL Standard. He has been published extensively and has written many technical reports. He was selected as one of the U.S. representatives for engineering educational and consultation mission to Asia in 1989.

NADER F. MIR received the B.Sc. degree (with honors) in electrical \& computer engineering in 1985 and the M.Sc. and Ph.D. degrees both in electrical engineering from Washington University in St. Louis, MO, in 1990 and 1994 respectively. He is currently a tenured Full Professor at the Electrical Engineering Department of San Jose State University, California. Prior to this position, he was an Associate Professor at this school, and Assistant Professor at University of Kentucky in Lexington. From 1994 to 1996, he was a research scientist at the Advanced Telecommunications Institute, Stevens Institute of Technology in New Jersey, working on design of advanced telecommunication networks. From 1990 to 1994 he was with the Computer and Communications Research Center at Washington University in St. Louis and worked as a research assistant on design and analysis of high-speed switching systems project. His research interests are: analysis of computer communication networks, design and analysis of switching systems, network design for wireless ad-hoc and sensor systems, and applications of digital integrated circuits in computer communications. He is a senior member of the IEEE and has served as the member of Technical Program Committee and Steering Committee of a number of major IEEE networking conferences such as WCNC, GLOBECOM, and ICC. Dr. Mir has published numerous refereed technical journal and conference papers, all in the field of communications and networking. He has published a book on video communication engineering, and another textbook published by Prentice Hall Publishing Co. entitled "Computer \& Communication Networks, Design and Analysis". Dr. Mir has received a number of prestigious national and university awards including the university teaching recognition award and research excellence award. $\mathrm{He}$ is also the recipient of the 2004 IASTED and also 2005 IASTED Outstanding Tutorial Presentation awards. Currently, he has several journal editorial positions such as: the Editorial Board Member of the International Journal of Internet Technology and Secured Transactions, the Editor of Journal of Computing and Information Technology, and the Associate Editor of IEEE Communication Magazine.

SARHAN M. MUSA received B.Sc. and M.Sc. degrees in electrical engineering from City College of New York, NY in 1996 and 1997 respectively, and a Ph.D. degree from City University of New York, NY in electrical engineering in 2001. He is currently assistant professor in the engineering technology department of Prairie View A\&M University, Texas. He is the director of Prairie View A\&M Networking Academy (PVNA) and senior researcher for the Center of Excellence for Communication Systems Technology Research (CECSTR), Texas His research interests are: DSL technology, IP telephony, networking security, wireless technology, queuing and traffic management, computing technology, and satellite networking. 